(7\%) silicosis, 1 sarcoidosis and 3 unclear. 61 of 102 (60\%) were referred with either possible work related airflow obstruction, asthma like symptoms at work or work related allergy symptoms. 16/61 (26\%) were confirmed as occupational asthma due to a sensitising agent, 10 (16\%) constitutional asthma, 20 (33\%) no lung disease, 1 (1.6\%) smoking related COPD, 5 (8\%) inducible laryngeal obstruction, 2 anxiety, $1(1.6 \%)$ Reactive Airways Dysfunction syndrome, 1 (1.6\%) Byssinosis, 1 (1.6\%) work related anaphylaxis, 1 (1.6\%) work related urticaria. 3 had asthma but the cause remained unclear. 20/61 (33\%) or 1 in 3 cases referred with possible work related airflow obstruction, asthma or allergy had a work related final diagnosis.

Conclusion The majority of cases seen are to determine causes of airflow obstruction/asthma like symptoms rather than interstitial lung disease in the tertiary setting. Most of these are found not to have asthma or a work related diagnosis. More cases of occupational asthma $(16+1$ cases $)$ were identified in total than asbestosis (13 cases); however asbestos related pleural disease was frequently identified (15 cases), making asbestos related disease the most common occupational related lung diagnosis. The likelihood of diagnosing occupational asthma due to a sensitising agent in this tertiary setting is 1 in 4.1 in 3 cases referred with possible interstitial lung disease had sufficient exposure and clinical/radiological evidence for a diagnosis of asbestosis, 1 in 14 (7\%) had silicosis.

\section{P227 A NOVEL CT SCORING SYSTEM DIFFERENTIATES ADMISSIONS SECONDARY TO EOSINOPHILIC FROM NON-EOSINOPHILIC ASTHMA}

${ }^{1} \mathrm{G}$ Hynes, ${ }^{2} \mathrm{M}$ Tsakok, ${ }^{1} \mathrm{R}$ Shrimanker, ${ }^{3} \mathrm{M}$ Bradicich, ${ }^{2} \mathrm{~V}$ St Noble, ${ }^{2} \mathrm{~F}$ Gleeson, ${ }^{1} \mathrm{P}$ Pavord. ${ }^{1}$ University of Oxford, Oxford, UK; ${ }^{2}$ Oxford University Hospitals NHS Foundation Trust, Oxford, UK; ${ }^{3}$ University of Pisa, Pisa, Italy

\subsection{6/thoraxjnl-2017-210983.369}

Introduction Post-mortem studies of patients who have died from asthma show that mucus plugging of the airways is a prominent feature. We have investigated whether this can be identified and quantified on CT scans taken at the time of a severe asthma attack and tested the hypothesis that mucus plugging is specific to attacks associated with a raised blood eosinophil count.

Methods We developed a scoring system based on features on CT scans of asthmatic patients potentially associated with mucus plugging (see Table 1). We used this scoring system to retrospectively score CT scans of 6 patients admitted to the John Radcliffe Hospital, Oxford, with acute attacks of asthma. CT scans were performed within three days of admission to investigate whether there was an alternative cause for the patients' presenting symptoms. Two radiologists, blinded to clinical measures for the patients, independently scored the CT scans.

Results Four patients had a blood eosinophil count $\geq 0.3 \times$ $10^{9} /$ litre at the time of admission. The mean (range) CT score was 18 (17) in these patients and 6 (4) in the non-eosinophilic patients. The intraclass correlation coefficient between the two radiologists' scores was 0.823 . The largest contributors to the difference between the eosinophilic and non- eosinophilic patients' scores was bronchial wall thickening (6 v 1) and mucus plugging ( $3 \mathrm{\vee} 0)$.

Conclusion Our scoring system was repeatable between observers and might potentially identify a pathophysiological mechanism particularly associated with eosinophilic asthma attacks.

Abstract P227 Table 1 CT scoring protocol for patients admitted secondary to asthma. Scans were interpreted by a specialist chest radiologist from slices at three locations on each lung: at the level of the inferior pulmonary vein, at the origin of the lingular bronchus, and at the aortic arch. This gives a score between 0 and 54.

\begin{tabular}{lllll}
\hline & 0 & 1 & 2 & 3 \\
\hline $\begin{array}{l}\text { Bronchial wall } \\
\text { thickening }\end{array}$ & None & Some & Prominent & \\
$\begin{array}{l}\text { Mucus plugging } \\
\text { Mosiacism }\end{array}$ & None & Some & Prominent & \\
Atelectasis & None & Minor & Major & \\
\hline
\end{tabular}

\section{P228 FATTY ACID SUPPLEMENTATION AND ASTHMA: A SYSTEMATIC REVIEW}

WF Kwok, AW Wilson. Norfolk and Norwich Hospital, Norwich, UK

\subsection{6/thoraxjnl-2017-210983.370}

Background Emerging evidence suggests that fatty acid supplementation has a direct effect on the inflammatory cascade, with recently discovered pro-resolving lipid mediators inducing anti-inflammatory mechanisms in animal studies. We aimed to conduct a systematic review to investigate the relationship between omega-3 fatty acid supplementation and respiratory outcomes in adult patients with asthma compared to nonsupplementation.

Methods We identified randomised controlled studies of fatty acid supplementation in adults with asthma through a systematic review of the databases Pubmed, Medline, Embase, CINAHL. Returned Results were screened, quality assessed and cross-checked according to the study inclusion criteria by two researchers.

Results The search found seven high quality studies in the literature suitable for inclusion in the review. Four studies did not show a significant difference in FEV1 between intervention and control groups. Two studies showed an improvement with bronchodilator use, two did not. Three studies showed improvement in Asthma symptoms compared to two studies of no benefit. Two studies showed improvement in peak flow, whereas two did not. Small patient numbers recruited in the studies, differences in fatty acid supplementation at different dosages and lack of published studies limit the strength of evidence presented.

Conclusion There is no significant evidence to suggest supplementation with fatty acids improve pulmonary function in adult patients with Asthma. Evidence for improvement in symptom control is unequivocal. Much of the data is from small short duration studies. Larger studies are required, with non-biologically active control, In the future to evaluate clinical correlation between supplementation and effect on asthma control. 


\section{Withdrawn: P228 Fatty acid supplementation and asthma: a} systematic review

Kwok WF, Wilson AW. P228 Fatty acid supplementation and asthma: a systematic review. Thorax 2017;72:A207. doi:10.1136/thoraxjnl-2017-210983.370

This abstract has been withdrawn. It was not presented at the meeting.

(C) Article author(s) (or their employer(s) unless otherwise stated in the text of the article) 2018. All rights reserved. No commercial use is permitted unless otherwise expressly granted.

Thorax 2018;73:360. doi:10.1136/thoraxjnl-2017-210983.370wthn

D) Check for updates 